\title{
Neutrophil to lymphocyte ratio predicts short- and long-term mortality following revascularization therapy for ST elevation myocardial infarction
}

\author{
Abhishek C. Sawant ${ }^{1}$, Prabhat Adhikari ${ }^{1}$, Swapna R. Narra ${ }^{1}$, \\ Shantanu V. Srivatsa ${ }^{1}$, Paul K. Mills ${ }^{2}$, Sanjay S. Srivatsa ${ }^{1}$ \\ ${ }^{1}$ Community Regional Medical Center, Fresno, California, USA \\ ${ }^{2}$ University of California at San Francisco, Fresno Medical Education Program, Fresno, California, USA
}

\begin{abstract}
Background: Several inflammation biomarkers have been implicated in the pathogenesis and prognosis of acute coronary syndromes. However, the prognostic role of the neutrophil-lymphocyte white cell interactive response to myocardial injury in predicting short-and long-term mortality after ST elevation myocardial infarction (STEMI) remains poorly defined.
\end{abstract}

Methods: We evaluated 250 consecutive STEMI patients presenting acutely for revascularization to our tertiary care center over 1 year. Patients with acute sepsis, trauma, recent surgery, autoimmune diseases, or underlying malignancy were excluded. Data gathered included demographics, clinical presentation, leukocyte markers, electrocardiograms, evaluations, therapy, major adverse cardiac events, and all-cause mortality.

Results: Mean age was $62 \pm 15$ years, $70.4 \%$ of subjects were males while majority (49.4\%) were Caucasians. Mean duration of follow-up was $571 \pm 291$ days (median 730 days). Univariate analysis of several inflammatory biomarkers including $C$-reactive protein, revealed white cell count $(O R=1.09, p<0.001)$ and neutrophil to lymphocyte ratio $(N L R)(O R=1.05$, $p=0.011)$ as predictors of short- and long-term mortality; but not mean neutrophil count $(O R=1.04, p=0.055)$ or lymphocyte count alone $(O R=0.96, p=0.551)$. Multivariate analysis using backward stepwise regression revealed $N L R(O R=2.64, p=0.026)$, female gender $(O R=5.35, p<0.001)$, cerebrovascular accident history $(O R=3.36, p=0.023)$, low glomerular filtration rate $(O R=0.98, p=0.012)$ and cardiac arrest on admission $(O R=17.43, p<0.001)$ as robust independent predictors of long-term mortality. NLR was divided into two sub-groups based on an optimal cut off value of 7.4. This provided the best discriminatory cut off point for predicting adverse mortality outcome. Both short-term ( $\leq 30$ days) and long-term ( $\leq 2$ years) mortality were predicted with Kaplan-Meier survival curve separation best stratified by a NLR cut off value of 7.4.

Conclusions: NLR based on an optimal cut off value of 7.4, was an excellent predictor of short- and long-term survival in patients with revascularized STEMI and warrants larger scale multi-center prospective evaluation, as a prognostic indicator. NLR offers improved prognostic capacity when combined with conventional clinical scoring systems, such as the Thrombolysis In Myocardial Infarction risk score. (Cardiol J 2014; 21, 5: 500-508)

Key words: neutrophil to lymphocyte ratio, ST elevation myocardial infarction, Thrombolysis In Myocardial Infarction risk score, predictors of mortality, percutaneous coronary revascularization

Address for correspondence: Sanjay S. Srivatsa, MD, FACC, FSCAI, FESC, 7215 North Fresno Street, Suite 103, Fresno, CA, 93720, USA, tel: (559)-438-1111, e-mail: sanjaysrivatsa@hotmail.com 


\section{Introduction}

Several researchers have shown an association between various biomarkers of inflammation and pathophysiology of acute coronary syndromes (ACS) $[1,2]$. Recently, multiple studies have shown that inflammatory biomarkers, e.g. white cell count and neutrophil to lymphocyte ratio (NLR), may have prognostic value in stable coronary artery disease (CAD), as well as in ACS [3, 4]. NLR is a surrogate marker for the combined effects of the innate immune response (substantially mediated by neutrophils), and the subsequent adaptive immune response (substantially mediated by lymphocytes). NLR may also reflect the myocardial remodeling responses after reperfusion injury [5-7]. Nevertheless, the role of NLR in predicting short- and long-term mortality after ST-segment elevation myocardial infarction (STEMI), treated with primary percutaneous coronary intervention $(\mathrm{PCI})$ in the drug eluting stent era remains undefined. Our study aimed to determine if NLR as an all-cause mortality outcome predictor, could be utilized alone, and in combination with current standardized cardiac risk score models, to improve the prediction of subsequent short- and long-term all-cause mortality.

\section{Methods}

Two hundred and seventy-eight consecutive patients presenting from January 2007 to January 2008 with a diagnosis of STEMI based on electrocardiographic (ECG) criteria were studied and followed until December 2010. Data prospectively collected in an ACS outcomes database were then retrospectively analyzed. Myocardial infarction (MI) was diagnosed by symptoms of ischemia and elevation in cardiac biomarkers, as defined by the Joint European Society of Cardiology and the American College of Cardiology [8, 9]. STEMI was defined as ST segment elevations measuring $0.2 \mathrm{mV}$ in leads $\mathrm{V} 1$ to $\mathrm{V} 3$ or $0.1 \mathrm{mV}$ in all other leads with the above-mentioned changes present in at least two contiguous leads. Twelve lead ECGs were obtained at $25 \mathrm{~mm} / \mathrm{s}$ paper speed, and $10 \mathrm{mV}$ gain. Analysis of the first presenting ECG included: heart rate (HR), PR interval, QRS duration, corrected QT interval, QRS axis and T-wave axis.

\section{Exclusion criteria}

Out of the cohort of 278 patients, 11 patients were excluded due to presence of left bundle branch block or atrial fibrillation on the presen- ting ECG. Five patients had a diagnosis of cancer; 4 patients had underlying sepsis on presentation while 2 patients had recently undergone cardiac surgery. In 6 patients a complete blood count was not available leaving 250 patients for analysis.

\section{Data collected}

At presentation 12 lead ECG, complete blood count, metabolic panel, radiographic investigations, and echocardiographic data were obtained. All patients underwent emergent coronary angiography, and based on angiographic and clinical findings were treated with percutaneous coronary revascularization or urgent coronary artery bypass grafting according to contemporaneous guidelines. Patients underwent concurrent anticoagulant therapy for PCI with unfractionated heparin/low molecular weight heparin and peri-procedural intravenous glycoprotein IIb/IIIa inhibitors, or bivalirudin (majority of cases). After coronary stent placement, patients received adjunctive dual antiplatelet therapy with aspirin and a thienopyridine antagonist (mostly clopidogrel). Clinical information abstracted included systemic hypertension, diabetes mellitus (DM), body mass index (BMI), prior $\mathrm{MI}$, prior congestive heart failure (CHF), prior coronary revascularization, smoking status and relevant family history. Thrombolysis In Myocardial Infarction (TIMI) study group definitions for current smoking and family history were utilized [10]. Congestive heart failure was defined as ejection fraction (EF) less than 35\%, elevated B-type natriuretic peptide (BNP) and/or evidence of fluid overload on chest radiograph. TIMI grade flow was measured as $0=$ no perfusion, $1=$ penetration without perfusion, 2 = partial reperfusion and $3=$ complete reperfusion [11].

\section{Blood sampling}

Blood sampling was performed within $24 \mathrm{~h}$ of STEMI presentation. A complete blood count with 6-part differential was obtained with an automated Sysmex XE 5000 Hematology System (Sysmex America Inc., Mundelein, IL, USA). Cardiac biomarkers including BNP and troponin I levels using an Advia Centaur XP Immunoassay (Siemens AG, Erlangen, Germany). Renal function, electrolytes and fasting lipid panel were measured using Advia 1800 Clinical Chemistry systems (Siemens AG, Erlangen, Germany).

\section{Outcome analysis}

The primary endpoint used for the analysis was all cause mortality at the end of 2 years. Ma- 
jor adverse cardiovascular events (MACE) data including cardiovascular death, $\mathrm{CHF}$, recurrent MI, and cardiac arrest were assessed at 30 days, 6 months and 2 years. Mortality data during the index admission were obtained from hospital records. Subsequent 6 months and 2 -year mortality data were obtained either from our hospital, or California Department of Public Health and Social Security Death Index records. Thrombolysis In Myocardial Infarction (TIMI-STEMI), Mayo Clinic Risk Score (MCRS) Global Registry of Acute Coronary Events (GRACE) scores were also calculated for all subjects [10, 12, 13].

This study was approved by the institutional review board of Community Regional Medical Centers.

\section{Statistical analysis}

Statistical analysis was performed using SPSS Software (PASW for Windows, Rel. 18.0.0. 2009; SPSS Inc., Chicago, IL, USA). Continuous variables were summarized as mean \pm standard deviation (SD) and comparisons between continuous variables utilized the Student's t-test. Categorical variables were summarized as percentages of the group total and comparisons between groups were analyzed using either Fisher exact test or $\chi^{2}$ where appropriate. NLR was utilized as both a continuous and categorical variable, based on relative risk of mortality. Assessment of the bivariate relationship between mortality and each risk factor was performed using data from 250 patients. Variables identified as significant ( $\mathrm{p}$ value $<0.1$ ) during univariate analysis were then fitted in a logistic regression model by a backward elimination method. This adjusted for confounders and enabled determination of variables of interest associated with increased risk of mortality or major cardiovascular adverse outcomes. Differences in survival curves obtained using Kaplan-Meier analysis were compared using the log-rank test. Receiver operating curves (ROC) were constructed to obtain area under the curve (AUC), and to predict cut off values of NLR that could be used to predict mortality. Statistical significance was accepted at $\mathrm{p}$ value $<0.05$.

\section{Results}

Patient demographics, relevant medical history, medications, laboratory findings, ECG data and treatments received are summarized in Table 1. Mean age was $62 \pm 15$ years, $70.4 \%$ of subjects were males while majority $(49.4 \%)$ were Caucasians. Mean duration of follow-up was $571 \pm$ \pm 291 days (median 730 days) and mean NLR was $6.60 \pm 7.8$. Age $(\mathrm{r}=0.198, \mathrm{n}=250, \mathrm{p}=0.002)$ and BNP level $(r=0.208, \mathrm{n}=127, \mathrm{p}=0.019)$ were positively correlated with NLR. Patients with higher NLR were more likely to be: older $(p=0.002)$, have CHF on presentation ( $\mathrm{p}=0.003)$; have elevated white cell count $(\mathrm{p}<0.001)$; prolonged QRS duration $(\mathrm{p}=0.040)$ and less likely to undergo PCI $(\mathrm{p}<0.001)$. Standardized risk assessment scores like (TIMI-STEMI, MCRS and GRACE) were correspondingly elevated in patients with higher NLR values.

\section{Predictive value of NLR}

NLR was divided into two sub-groups based on optimal cut off value of 7.4 , which provided the highest predictive power for mortality. NLR values of $\geq 7.4$ demonstrated a sensitivity of $47.5 \%$ (95\% CI $34.3-60.9 \%$ ), specificity of $81.2 \%$ (95\% CI 74.9-86.4\%), and positive likelihood ratio of 2.52 (95\% CI 1.69-3.75) for mortality. ROC curves were constructed to assess the predictive value of NLR. To predict long-term mortality using NLR prediction ROC was performed to obtain AUC with $\mathrm{C}$-statistic of 0.73 . In order to improve the predictive power of NLR in point of care scenarios, the NLR was combined with standardized 'TIMI risk score for STEMI' variables. The predictive power was significantly improved when NLR was added to TIMI score clinical variables by assigning one point for NLR $\geq 7.4$. Using this approach, the ROC AUC mortality prediction improved significantly to a C-statistic of 0.80, p < 0.001 (Fig. 1).

\section{Outcomes}

Mortality outcomes stratified by NLR cut off value of 7.4, at 3 time intervals (in-hospital and up to 30 days post STEMI, 30 days to 6 months, 6 months to $\leq 2$ years) are shown in Figure 2. Compared to NLR $<7.4$, patients with NLR $\geq 7.4$ had significantly higher mortality at all 3 follow-up time points: immediate hospitalization period, short-term and long-term. In the first 30 days after hospitalization, a total number of 41 deaths were observed, while a total number of 59 deaths were observed at the end of 2 years. The prediction of mortality at these 3 time points is of considerable clinical significance, due to the varying spectrum of modifiable risk factors at differing post STEMI time points. Table 2 outlines the MACE endpoints measured during index hospitalization and up to 2 years of follow-up. The achievement of post-procedural TIMI grade 3 flow after PCI, did not differ significantly between patients with NLR 
Table 1. Patient characteristics including demographics, medical history, medications, laboratory/electrocardiography variables, anticoagulant therapy, reperfusion modality, and clinical risk scores stratified by neutrophil to lymphocyte ratio cut off value of 7.4.

\begin{tabular}{|c|c|c|c|c|}
\hline & $\begin{array}{c}\text { All } \\
(n=250)\end{array}$ & $\begin{array}{c}\text { NLR }<7.4 \times 10^{9} / L \\
(n=186)\end{array}$ & $\begin{array}{c}\text { NLR } \\
(n=64)\end{array}$ & $\mathbf{P}$ \\
\hline \multicolumn{5}{|l|}{ Demographics } \\
\hline Age & $62 \pm 15$ & $60 \pm 14$ & $67 \pm 15$ & 0.002 \\
\hline Gender (male) & $176(70.4 \%)$ & $132(71 \%)$ & $44(68.8 \%)$ & 0.737 \\
\hline Race (Caucasian) & $123(49.4 \%)$ & $88(47.6 \%)$ & $35(54.7 \%)$ & 0.368 \\
\hline \multicolumn{5}{|l|}{ Medical history } \\
\hline Smoking & $114(45.6 \%)$ & $82(44.1 \%)$ & $32(50 \%)$ & 0.413 \\
\hline Diabetes & $68(27.4 \%)$ & $52(28.3 \%)$ & $15(25 \%)$ & 0.614 \\
\hline Hypertension & $179(71.9 \%)$ & $130(70.3 \%)$ & $49(76.6 \%)$ & 0.334 \\
\hline Congestive heart failure & $70(28 \%)$ & $43(23.1 \%)$ & $27(42.2 \%)$ & 0.003 \\
\hline Atrial fibrillation & $47(18.9 \%)$ & $34(18.4 \%)$ & $13(20.3 \%)$ & 0.733 \\
\hline Renal disease & $26(10.4 \%)$ & $17(9.1 \%)$ & $9(14.1 \%)$ & 0.266 \\
\hline \multicolumn{5}{|l|}{ Medications } \\
\hline Aspirin use & $83(33.6 \%)$ & $61(33.2 \%)$ & $22(34.9 \%)$ & 0.798 \\
\hline Beta-blocker use & $79(32.1 \%)$ & $62(33.9 \%)$ & $17(27 \%)$ & 0.312 \\
\hline Statin use & $83(33.6 \%)$ & $61(33.2 \%)$ & $22(34.9 \%)$ & 0.798 \\
\hline Angiotensin converting enzyme inhibitor use & $92(37.2 \%)$ & $69(37.5 \%)$ & $23(36.5 \%)$ & 0.888 \\
\hline \multicolumn{5}{|l|}{ Presenting laboratory findings } \\
\hline Troponin on admission & $29.59 \pm 122$ & $19.27 \pm 76.39$ & $57.69 \pm 201.22$ & 0.148 \\
\hline B-type natriuretic peptide & $946 \pm 1314$ & $862 \pm 1336$ & $1130 \pm 1263$ & 0.288 \\
\hline White cell count & $11.9 \pm 6.1$ & $11.08 \pm 5.45$ & $14.68 \pm 7.48$ & $<0.001$ \\
\hline Neutrophil count & $8.7 \pm 6.65$ & $7.43 \pm 6.30$ & $12.41 \pm 6.28$ & $<0.001$ \\
\hline Lymphocyte count & $2.26 \pm 2.29$ & $2.69 \pm 2.49$ & $0.98 \pm 0.52$ & $<0.001$ \\
\hline Hematocrit & $40.8 \pm 6.4$ & $41.84 \pm 5.60$ & $37.84 \pm 7.84$ & 0.001 \\
\hline \multicolumn{5}{|l|}{ Electrocardiogram changes } \\
\hline Heart rate & $83.66 \pm 26.47$ & $82.04 \pm 24.4$ & $88.78 \pm 31.77$ & 0.080 \\
\hline QRS duration & $96.63 \pm 24.67$ & $94.53 \pm 22.61$ & $102.83 \pm 29.74$ & 0.044 \\
\hline Corrected QT interval & $426 \pm 39.67$ & $425.4 \pm 38.86$ & $427.9 \pm 43.14$ & 0.666 \\
\hline ST segment resolution & $168(67.5 \%)$ & $131(70.8 \%)$ & $37(57.8 \%)$ & 0.056 \\
\hline \multicolumn{5}{|l|}{ Treatment } \\
\hline Heparin (unfractionated) & $60(24.2 \%)$ & $43(23.4 \%)$ & $17(26.6 \%)$ & 0.607 \\
\hline Low molecular weight heparin & $40(16.1 \%)$ & $31(16.8 \%)$ & $9(14.1 \%)$ & 0.602 \\
\hline Percutaneous coronary intervention & $157(63.1 \%)$ & $128(69.2 \%)$ & $29(45.3 \%)$ & 0.001 \\
\hline Coronary artery bypass grafting & $34(13.7 \%)$ & $26(14 \%)$ & $8(12.7 \%)$ & 0.798 \\
\hline \multicolumn{5}{|l|}{ Risk scores for mortality } \\
\hline TIMI-STEMI & $4.55 \pm 2.87$ & $4.16 \pm 2.79$ & $5.7 \pm 2.75$ & $<0.001$ \\
\hline Mayo Clinic Risk Score & $8.25 \pm 7.45$ & $7.28 \pm 6.42$ & $11.38 \pm 9.38$ & $<0.001$ \\
\hline Global Registry of Acute Coronary Events ${ }^{\odot}$ & $125.8 \pm 45.66$ & $117.27 \pm 42.68$ & $150.78 \pm 45.09$ & $<0.001$ \\
\hline
\end{tabular}

TIMI-STEMI — Thrombolysis in Myocardial Infarction-ST elevation myocardial infarction

$<7.4$, as compared to patients with NLR $\geq 7.4$. NLR was also not significantly different between patients who achieved TIMI 0 flow compared to TIMI grade 2 or 3 flow after PCI.

\section{Regression analysis}

Using all-cause mortality as the primary outcome, univariate analysis was performed to determine which variables predicted mortality. Female gender,
Hispanic race, age, BMI, smoking status, CHF history, cerebrovascular accident history, Killip CHF class on admission, high white cell count, low hemoglobin, elevated BNP, low glomerular filtration rate (GFR) and cardiac arrest on presentation were all associated with elevated mortality risk (Table 3 ). When these variables were included in a multivariate model using backward stepwise regression, NLR $(\mathrm{OR}=2.64, \mathrm{p}=0.026)$, female gender $(\mathrm{OR}=5.35$, 


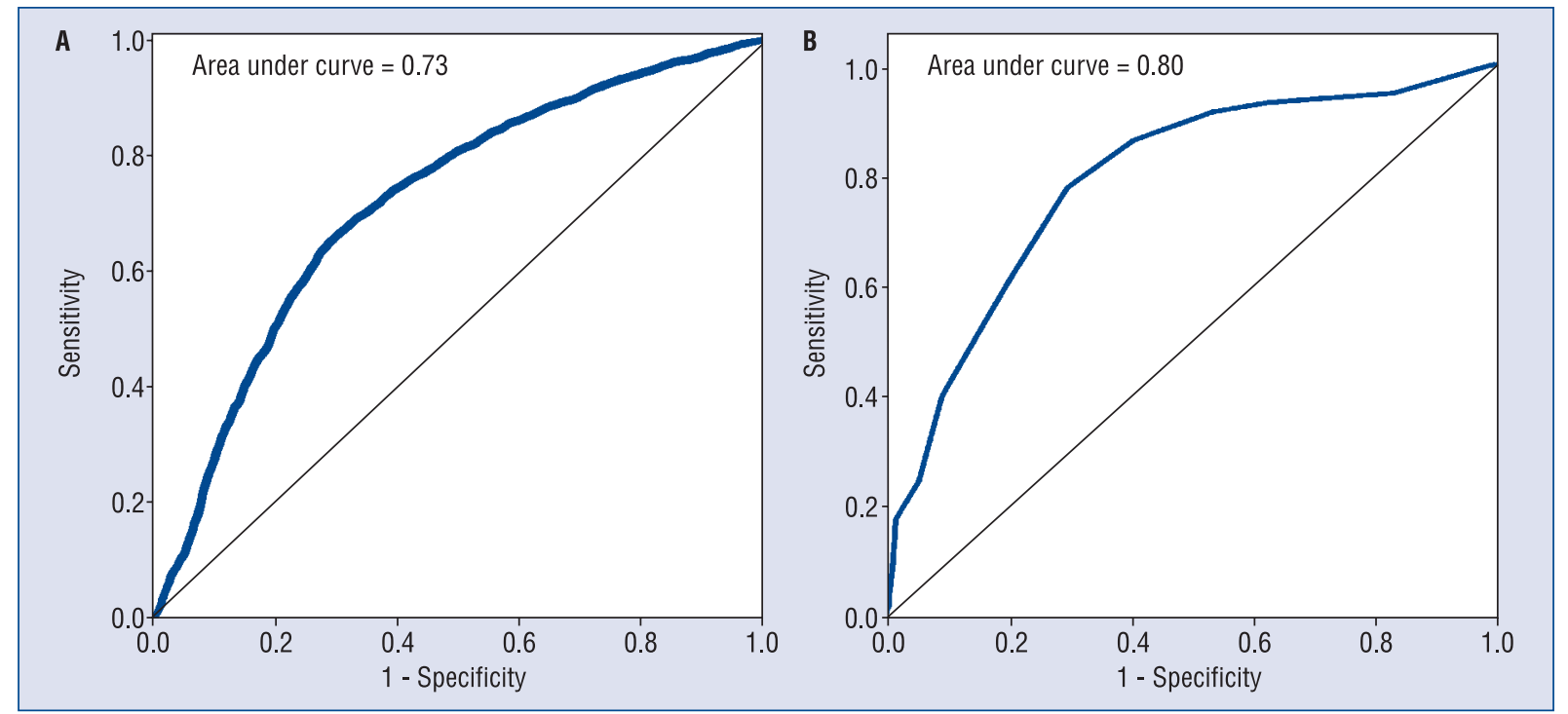

Figure 1. A. Receiver operating characteristic (ROC), area under the curve (AUC) analysis of neutrophil to lymphocyte ratio (NLR) alone in prediction of post-ST elevation myocardial infarction (STEMI) revascularization mortality; B. Significant improvement in predictive power of Thrombolysis in Myocardial Infarction-STEMI risk score variables by addition of NLR variable: c-statistic AUC improvement from 0.73 to 0.80 ( $p<0.001$ ).

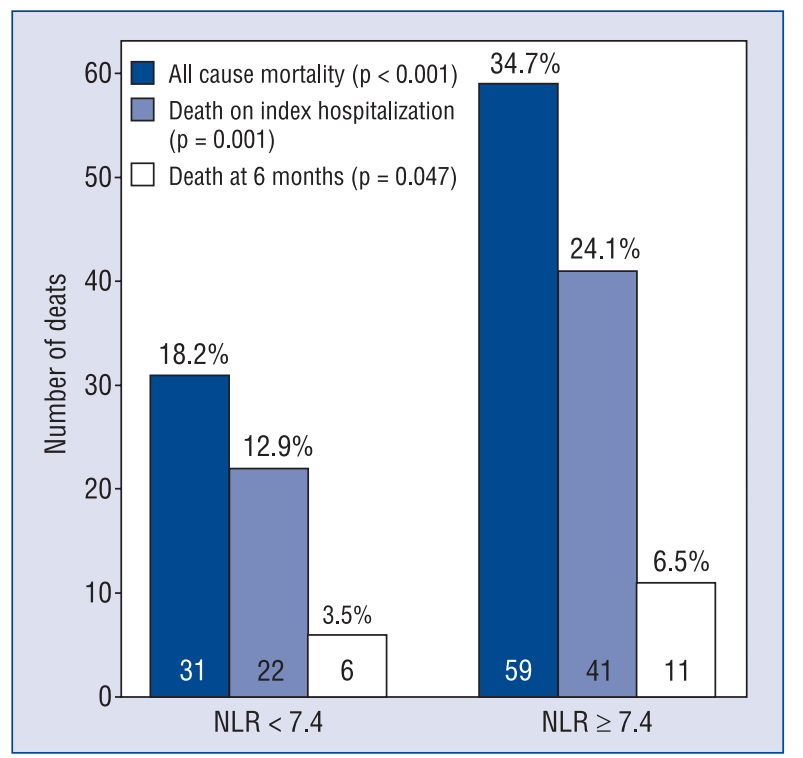

Figure 2. Stratification of all-cause mortality based on neutrophil to lymphocyte ratio (NLR) cut off value of 7.4 into 3 time periods: in-hospital and up to 30 days, 30 days to 6 months, and 6 months to $\leq 2$ years mortality rates.

$\mathrm{p}<0.001)$, cerebrovascular accident history $(\mathrm{OR}=3.36, \mathrm{p}=0.023)$, low $\mathrm{GFR}(\mathrm{OR}=0.98$, $\mathrm{p}=0.012)$, and cardiac arrest on admission $(\mathrm{OR}=17.43, \mathrm{p}<0.001)$ remained as independent predictors of long-term mortality as illustrated by the Forest plot (Fig. 3).
Table 2. Major adverse cardiac event (MACE) end point frequencies up to 2-year follow-up.

\begin{tabular}{lc}
\hline MACE endpoints at 2 years & All patients \\
\hline Recurrent myocardial infarction & $28(11.2 \%)$ \\
Cardiac death & $34(13.6 \%)$ \\
Congestive heart failure & $87(34.8 \%)$ \\
Cerebrovascular accident & $6(2.4 \%)$ \\
Repeat coronary artery bypass graft & $4(1.6 \%)$ \\
Repeat percutaneous coronary & $5(2 \%)$ \\
intervention & \\
\hline
\end{tabular}

\section{Survival analysis}

Kaplan-Meier survival analysis was performed to measure all-cause mortality and survival free from systolic heart failure, stratified by NLR. Patients with NLR $<7.4$ had higher survival compared to patients whose NLR was $\geq 7.4 \mathrm{du}-$ ring short-term follow-up of 30 days (Fig. 4A) and long-term follow-up of 2 years (Fig. 4B). NLR was also found to be a strong predictor of survival free from systolic heart failure $(\mathrm{EF}<35 \%)$ as shown in Figure 4C.

\section{Discussion}

This study has demonstrated that in patients presenting for primary PCI revascularization with STEMI, an elevated NLR (based on an optimum 
Table 3. Univariate predictors of long-term mortality following revascularized ST elevation myocardial infarction.

\begin{tabular}{|c|c|c|}
\hline \multirow[t]{2}{*}{ Variable } & \multicolumn{2}{|c|}{ Mortality in 1 year $(n=59 / 250)$} \\
\hline & $95 \%$ confidence interval & $\mathbf{P}$ \\
\hline Gender: male $=$ reference & $3.95(2.15-7.25)$ & $<0.001$ \\
\hline Race: Caucasian = reference & $3.6(1.32-9.8)$ & 0.012 \\
\hline Age & $1.06(1.04-1.08)$ & $<0.001$ \\
\hline Body mass index & $0.90(0.85-0.95)$ & $<0.001$ \\
\hline Diabetes type 2 & $1.30(0.69-2.44)$ & 0.416 \\
\hline Smoker & $2.87(1.52-5.42)$ & 0.001 \\
\hline Hypertension & $1.32(0.67-2.59)$ & 0.442 \\
\hline Hyperlipidemia & $1.45(0.81-2.60)$ & 0.213 \\
\hline Congestive heart failure & $3.43(1.86-6.31)$ & $<0.001$ \\
\hline Atrial fibrillation & $1.17(0.57-2.44)$ & 0.667 \\
\hline Previous myocardial infarction & $1.32(0.72-2.41)$ & 0.369 \\
\hline Cerebrovascular accident & $4.10(1.86-9.0)$ & $<0.001$ \\
\hline Killip (Class 1 = reference) & $7.46(2.85-19.52)$ & $<0.001$ \\
\hline Troponin at admit & $1.00(0.99-1.00)$ & 0.644 \\
\hline White cell count & $1.09(1.04-1.1)$ & $<0.001$ \\
\hline Neutrophil count & $1.04(0.99-1.09)$ & 0.055 \\
\hline Lymphocyte count & $0.96(0.83-1.10)$ & 0.551 \\
\hline Neutrophil to lymphocyte ratio & $1.05(1.01-1.08)$ & 0.011 \\
\hline Anemia (hematocrit < 36) & $5.61(2.34-13.43)$ & $<0.001$ \\
\hline Glomerular filtration rate & $0.98(0.97-0.99)$ & $<0.001$ \\
\hline B-type natriuretic peptide & $1.01(1.00-1.01)$ & 0.003 \\
\hline Left ventricular hypertrophy & $1.19(0.57-2.50)$ & 0.646 \\
\hline Heart rate & $1.09(0.99-1.02)$ & 0.086 \\
\hline PR interval & $1.00(0.96-1.00)$ & 0.939 \\
\hline QRS duration & $1.01(0.99-1.02)$ & 0.081 \\
\hline Corrected QT Interval & $1.00(0.996-1.01)$ & 0.392 \\
\hline T wave changes & $2.81(1.20-6.56)$ & 0.017 \\
\hline ST segment elevation & $0.64(0.35-1.16)$ & 0.139 \\
\hline Cardiac arrest on admit & $14.83(7.30-30.12)$ & $<0.001$ \\
\hline TIMI grade 3 flow after $\mathrm{PCI}$ & $1.17(0.59-2.32)$ & 0.645 \\
\hline MVD ( $\geq 2$ vessel involved) & $2.94(1.23-7.03)$ & 0.015 \\
\hline
\end{tabular}

$\mathrm{PCl}$ - percutaneous coronary intervention; TIMI — Thrombolysis in Myocardial Infarction MVD — multivessel disease

derived cut off value $>7.4$ ) is independently associated with higher all-cause mortality and higher MACE rates at both short-term ( $\leq 30$ days), and long-term ( $\leq 2$ years) follow-up. Rapid separation of Kaplan-Meier survival curves was observed within 1-week post-STEMI PCI. An elevated NLR reflects both neutrophilia and relative lymphopenia. Several studies have examined the role of neutrophils and lymphocytes in modulating the inflammatory response to myocardial injury [14-16]. Neutrophils are speculated to mediate increased plaque rupture and thrombosis by secreting proteolytic enzymes causing vascular damage, activation of coagulation pathways, micro vascular plugging and myocyte necrosis mediated by secretion of pro-inflammatory cytokines [1, 17]. Activated leukocytes may also modulate the electrical activity of the myocardium (and therefore arrhythmogenesis), by release of oxygen free radicals [18]. Leukocyte count has an interplay with other known CAD risk factors, such that it enhances the diagnostic capacity of conventional risk factors in predicting CAD [19]. Physiological stress and the subsequent activation of the neurohormonal system during STEMI leads to cortisol release, which in turn mediates lymphopenia through apoptosis [20]. Atherosclerosis and plaque rupture leading to ACS is an inflammatory process mediated by the complex interplay 


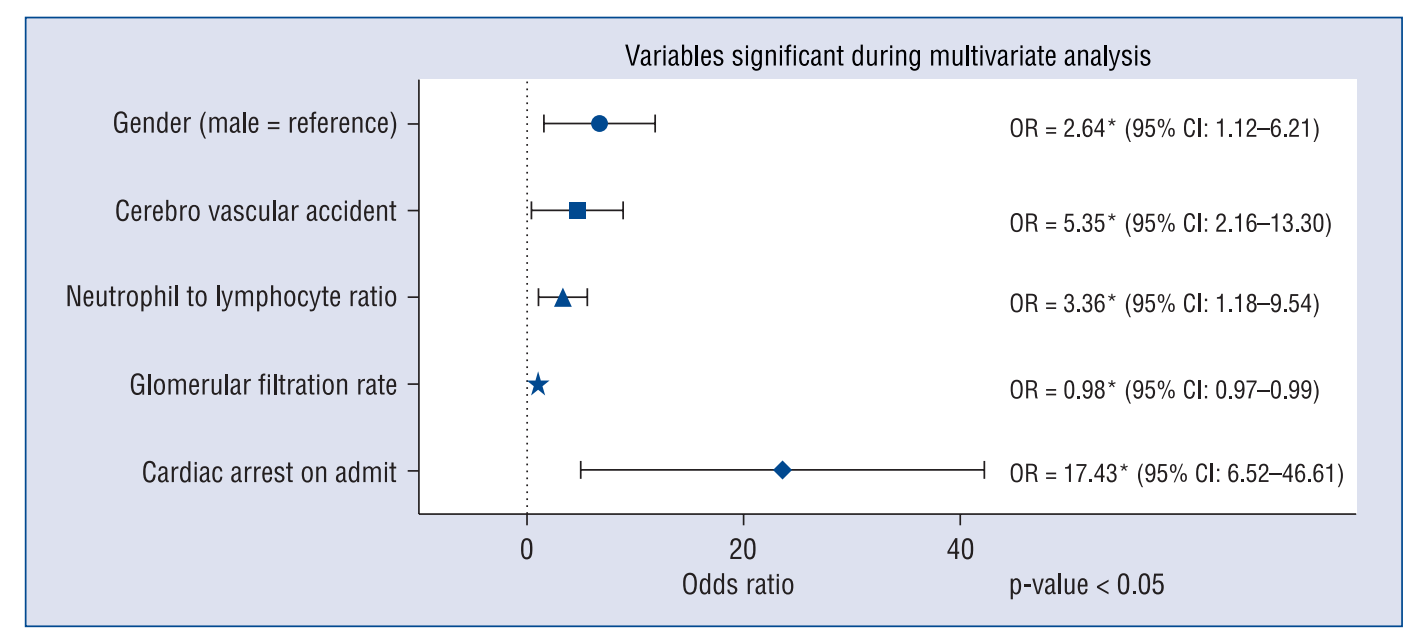

Figure 3. Multivariate analysis of long-term mortality risk factors; ${ }^{*} p<0.05$.
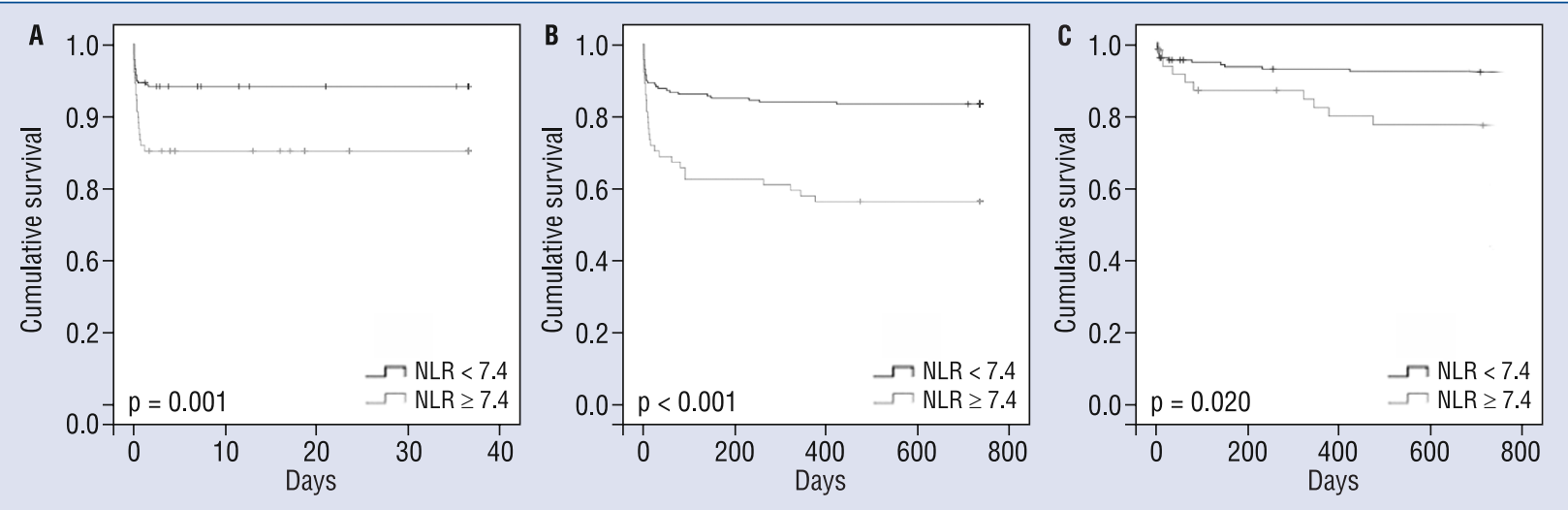

Figure 4. Kaplan-Meier survival curve analysis of all-cause mortality for short-term (A), long-term (B) end points stratified by neutrophil to lymphocyte ratio (NLR) cut off value of 7.4. Kaplan-Meier survival curve analysis of survival free of heart failure (C) stratified by NLR cut off value of 7.4.

between the innate neutrophil mediated reactive immune responses, and subsequent lymphocyte mediated adaptive immune responses [5-7]. Thus, NLR may act as a combined surrogate marker for both the reactive and adaptive components of the inflammatory response that result in plaque rupture, ischemic myocardial damage, adverse ventricular remodeling, and consequent left ventricular dysfunction.

Older age is a major predictor of adverse outcome in ACS. Age $>70$ years has remained a major independent predictor of in-hospital cardiovascular mortality in many STEMI prognosis studies [21]. Similar to our cohort where NLR > 7.4 was independently associated with systolic heart failure, studies have shown that increased neu- trophil count has been reliably associated with increased MI size and decreased left ventricular function [22]. Evidence of $\mathrm{CHF}$ with elevated BNP levels is associated with poor prognosis due to increased myocardial damage [23]. This is the proposed physiologic basis for the observed correlation between increased NLR, older age, increased BNP levels, and diminished long-term survival. Consistent with this, we also observed that prolonged $\mathrm{QRS}$ duration was associated with elevated NLR values, and diminished survival in our study. A post-hoc analysis of the Efficacy of Vasopressin Antagonism in Heart Failure Outcome Study with Tolvaptan (EVEREST) showed similar findings, where lymphopenia was associated with widened QRS duration and older age (both markers 
of worse prognosis) [24]. Prolonged QRS reflects more extensive myocardial damage, and the adverse ventricular remodeling that accompanies this (vide supra).

We tested the predictive value of NLR both independently and in conjunction with well-validated standardized MI risk scores. We have shown that not only does NLR independently predict shortand long-term mortality; it significantly improves prediction of short- and long-term mortality, when incorporated as a co-variable in standardized risk scores (e.g. TIMI-STEMI risk score). Multiple ACS studies now support the use of NLR as an admission biomarker, which can be used to determine prognosis $[25,26]$. NLR can be readily calculated at point of care, thereby facilitating short- and long-term risk prediction for STEMI patients, even prior to revascularization. Arbel et al. [27] found that increased NLR was associated with increased severity of $\mathrm{CAD}$, thereby providing additive predictive value to conventional risk factors and commonly used biomarkers e.g. C-reactive protein and total white cell count. Similarly, in a large population based acute MI incident cohort, the absolute neutrophil count at presentation was strongly, positively, and independently associated with death/heart failure post-MI with an incremental discriminatory value for the absolute neutrophil count over traditional cardiac risk factors [28].

Female gender and low GFR has been shown in this study and others, to be independent predictors of mortality and MACE $[23,27,28]$. Our data was prospectively collected with NLR blood samples analyzed at admission, which is the most critical time point for clinicians to determine the choice of reperfusion therapy, based on the predicted interventional outcome of the presenting case. Pre-admission or initial presentation prognostic indices are of vital importance in the accurate triage of emergency room patients for STEMI revascularization. They may even be more important for the long-term prognosis of all-cause mortality, than the immediate outcome of PCI [25]. For instance, the overwhelmingly poor short- and long-term predicted mortality of certain STEMI patients, might preclude invasive intervention, and mandate more conservative management strategies. Interestingly, more complicated risk scoring systems that incorporate more comprehensive angiographic parameters and/or presentation hemodynamic variables, do not necessarily improve the long-term prediction of all-cause mortality [26]. Furthermore, the clinical variables used in risk prediction models vary in predictive risk assessment capacity accor- ding to the time point being considered post-MI $[29,30]$. In this regard, we suggest that the NLR is robust in its consistent benefit for reliably predicting both immediate, intermediate, and long-term mortality following revascularized STEMI.

\section{Limitations of the study}

Our study has several inherent limitations since retrospective analyses are subject to selection bias. Moreover, the population studied was from a single tertiary care center. However, our study population demographics reveal a good mix of Hispanic, African American and Asian populations (51.6\%) compared to the predominant Caucasian dominated populations studied in most major ACS clinical score derivation trials. While a single baseline admission complete blood count sampling has the benefit of being readily available, serial sampling may potentially yield a better analytical time point. However, since the exact time to peak inflammatory response after STEMI remains unknown, it is difficult to determine the most efficient collection time. Alternative concomitant etiologies for elevated NLR may have been present and not accounted for e.g. occult infection or malignancy. An NLR of 7.4 or greater at admission carried greater risk of adverse outcome in our study, although other studies have suggested differing NLR cut off points. A larger multi-center study with larger population size and diversity is warranted to best determine the future prognostic role of NLR, its best predictive cut off value, and sampling time.

\section{Conclusions}

NLR is a readily ascertainable, inexpensive, and reproducible biomarker for STEMI prognosis after PCI. It can be utilized as a robust stand-alone prognostic indicator for patients presenting with STEMI, even before eventual angiographic findings and outcomes are accounted for. When NLR is combined with standardized clinical mortality risk prediction scores, it markedly and significantly augments the predictive power of these scores.

\section{Acknowledgements}

The authors would like to acknowledge Judith Kammerer, Medical Librarian at Community Regional Medical Center (Fresno, California, USA) for help in obtaining research articles and Hwoi Chan Kwon, Pacific Union College (Angwin, California, USA) for collecting data during patient interviews.

Conflict of interest: None declared 


\section{References}

1. Nijm J, Wikby A, Tompa A, Olsson AG, Jonasson L. Circulating levels of proinflammatory cytokines and neutrophil-platelet aggregates in patients with coronary artery disease. Am J Cardiol, 2005; 95: 452-456.

2. Nessler J, Nessler B, Golebiowska-Wiatrak R et al. Serum biomarkers and clinical outcomes in heart failure patients treated de novo with carvedilol. Cardiol J, 2013; 20: 144-151.

3. Tamhane UU, Aneja S, Montgomery D, Rogers EK, Eagle KA, Gurm HS. Association between admission neutrophil to lymphocyte ratio and outcomes in patients with acute coronary syndrome. Am J Cardiol, 2008; 102: 653-657.

4. Ates AH, Canpolat $\mathrm{U}$, Yorgun $\mathrm{H}$ et al. Total white blood cell count is associated with the presence, severity and extent of coronary atherosclerosis detected by dual-source multislice computed tomographic coronary angiography. Cardiol J, 2011; 18: 371-377.

5. Mazzone A, De Servi S, Ricevuti G et al. Increased expression of neutrophil and monocyte adhesion molecules in unstable coronary artery disease. Circulation, 1993; 88: 358-363.

6. Ishikawa M, Vowinkel T, Stokes KY et al. CD40/CD40 ligand signaling in mouse cerebral microvasculature after focal ischemia/ /reperfusion. Circulation, 2005; 111: 1690-1696.

7. Huang Y, Rabb H, Womer KL. Ischemia-reperfusion and immediate T cell responses. Cell Immunol, 2007; 248: 4-11.

8. Myocardial infarction redefined--a consensus document of The Joint European Society of Cardiology/American College of Cardiology Committee for the redefinition of myocardial infarction. Eur Heart J, 2000; 21: 1502-1513.

9. Thygesen K, Alpert JS, White HD, Joint ESCAAHAWHFTFftRoMI. Universal definition of myocardial infarction. Eur Heart J, 2007; 28: 2525-2538.

10. Morrow DA, Antman EM, Charlesworth A et al. TIMI risk score for ST-elevation myocardial infarction: A convenient, bedside, clinical score for risk assessment at presentation: An intravenous $\mathrm{nPA}$ for treatment of infarcting myocardium early II trial substudy. Circulation, 2000; 102: 2031-2037.

11. Gibson CM, Cannon CP, Daley WL et al. TIMI frame count: A quantitative method of assessing coronary artery flow. Circulation, 1996; 93: 879-888.

12. Granger C.B GRJ, Dabbous O, et al. Predictors of Hospital Mortality in the Global Registry of Acute Coronary Events. Arch Intern Med, 2003; 163: 2345-2353.

13. Singh M, Gersh BJ, Li S et al. Mayo Clinic Risk Score for percutaneous coronary intervention predicts in-hospital mortality in patients undergoing coronary artery bypass graft surgery. Circulation, 2008; 117: 356-362.

14. Duffy BK, Gurm HS, Rajagopal V, Gupta R, Ellis SG, Bhatt DL. Usefulness of an elevated neutrophil to lymphocyte ratio in predicting long-term mortality after percutaneous coronary intervention. Am J Cardiol, 2006; 97: 993-996.

15. Anderson JL, Ronnow BS, Horne BD et al. Usefulness of a complete blood count-derived risk score to predict incident mortality in patients with suspected cardiovascular disease. Am J Cardiol, 2007; 99: 169-174.

16. Nunez J, Nunez E, Bodi $\mathrm{V}$ et al. Usefulness of the neutrophil to lymphocyte ratio in predicting long-term mortality in ST seg- ment elevation myocardial infarction. Am J Cardiol, 2008; 101: 747-752.

17. Manten A, de Winter RJ, Minnema MC et al. Procoagulant and proinflammatory activity in acute coronary syndromes. Cardiovasc Res, 1998; 40: 389-395.

18. Lucchesi BR, Werns SW, Fantone JC. The role of the neutrophil and free radicals in ischemic myocardial injury. J Mol Cell Cardiol, 1989; 21: 1241-1251.

19. Rasouli M, Nesarhosseini V, Kiasari AM et al. The multiplicative interactions of leukocyte counts with some other risk factors enhance the prognostic value for coronary artery disease. Cardiol J, 2011; 18: 246-253.

20. Onsrud M, Thorsby E. Influence of in vivo hydrocortisone on some human blood lymphocyte subpopulations. I. Effect on natural killer cell activity. Scand J Immunol, 1981; 13: 573-579.

21. Ergelen M, Uyarel H, Altay S et al. Predictive Value of Elevated Neutrophil to Lymphocyte Ratio in Patients Undergoing Primary Angioplasty for ST-Segment Elevation Myocardial Infarction. Clin Appl Thromb Hemost 2013; Jan 11, doi: 10.1177/1076029612473516 [E-pub ahead of print].

22. Chia S, Nagurney JT, Brown DF et al. Association of leukocyte and neutrophil counts with infarct size, left ventricular function and outcomes after percutaneous coronary intervention for ST-elevation myocardial infarction. Am J Cardiol, 2009; 103: 333-337.

23. de la Camara AG, Guerravales JM, Tapia PM et al. Role of biological and non biological factors in congestive heart failure mortality: PREDICE-SCORE: A clinical prediction rule. Cardiol J, 2012; 19: 578-585.

24. Vaduganathan MAA, Greene SJ, Mentz RJ et al; EVEREST trial investigators. Predictive value of low relative lymphocyte count in patients hospitalized for heart failure with reduced ejection fraction: insights from the EVEREST trial. Circ Heart Fail, 2012; 5: 750-758.

25. D'Ascenzo F, Biondi-Zoccai G, Moretti C et al. TIMI, GRACE and alternative risk scores in Acute Coronary Syndromes: A meta-analysis of 40 derivation studies on 216,552 patients and of 42 validation studies on 31,625 patients. Contemp Clin Trials, 2012; 33: 507-514.

26. Brkovic V, Dobric M, Beleslin B et al. Additive prognostic value of the SYNTAX score over GRACE, TIMI, ZWOLLE, CADILLAC and PAMI risk scores in patients with acute ST-segment elevation myocardial infarction treated by primary percutaneous coronary intervention. Int J Cardiovasc Imaging, 2013; 29: 1215-1228.

27. Arbel YFA, Halkin A, Birati EY et al.. Neutrophil/lymphocyte ratio is related to the severity of coronary artery disease and clinical outcome in patients undergoing angiography. Atherosclerosis, 2012; 225: 456-460.

28. Arruda-Olson AM, Reeder GS, Bell MR, Weston SA, Roger VL. Neutrophilia predicts death and heart failure after myocardial infarction: A community-based study. Circ Cardiovasc Qual Outcomes, 2009; 2: 656-662.

29. Amin ST, Morrow DA, Braunwald E et al. Dynamic TIMI risk score for STEMI. J Am Heart Assoc 2013; Jan 29, doi: 10.1161/ JAHA.112.003269 [E-pub ahead of print].

30. Chang WC, Kaul P, Fu Y et al. Forecasting mortality: Dynamic assessment of risk in ST-segment elevation acute myocardial infarction. Eur Heart J, 2006; 27: 419-426. 